\title{
Education on cardiac risk and CPR in cardiology clinic waiting rooms: a randomised clinical trial
}

\author{
Daniel McIntyre @ (1) 'Aravinda Thiagalingam, ${ }^{1,2}$ Harry Klimis, ${ }^{1}$ Amy Von Huben, ${ }^{1}$ \\ Simone Marschner (1), ${ }^{1}$ Clara K Chow (1) ${ }^{1,2}$
}

\begin{abstract}
- Additional supplemental material is published online only. To view, please visit the journal online (http://dx.doi. org/10.1136/heartjnl-2021319290)
\end{abstract}

'Westmead Applied Research Centre, The University of Sydney, Westmead, New South Wales, Australia

${ }^{2}$ Department of Cardiology, Westmead Hospital, Westmead, New South Wales, Australia

\section{Correspondence to}

Daniel McIntyre, The University of Sydney Westmead Applied Research Centre, Westmead, New South Wales, Australia; daniel.mcintyre@sydney.edu.au

Received 3 March 2021 Accepted 25 June 2021 Published Online First 21 July 2021

\section{SLinked}

- http://dx.doi.org/10.1136/ heartjnl-2021-319756

Check for updates

(C) Author(s) (or their employer(s)) 2021. No commercial re-use. See rights and permissions. Published by BMJ.

To cite: Mclntyre $D$,

Thiagalingam $A$,

Klimis $\mathrm{H}$, et al. Heart

2021:107:1637-1643.

\section{ABSTRACT \\ Objective Waiting time is inevitable during}

cardiovascular (CV) care. This study examines whether waiting room-based CV education could complement CV care.

Methods A 2:1 randomised clinical trial of patients in waiting rooms of hospital cardiology clinics. Intervention participants received a series of tablet-delivered CV educational videos and were randomised 1:1 to receive another video on cardiopulmonary resuscitation (CPR) or no extra video. Control received usual care. The primary outcome was the proportion of participants reporting high motivation to improve $\mathrm{CV}$ risk-modifying behaviours (physical activity, diet and blood pressure monitoring) post-clinic. Secondary outcomes: clinic satisfaction, CV lifestyle risk factors (RFs) and confidence to perform CPR. Assessors were blinded to treatment allocation.

Results Among 514 screened, 330 were randomised ( $n=220$ intervention, $n=110$ control) between December 2018 and March 2020, mean age 53.8 (SD 15.2), 55.2\% male. Post-clinic, more intervention participants reported high motivation to improve CV risk-modifying behaviours: $29.6 \%(64 / 216)$ versus $18.7 \%$ (20/107), relative risk (RR) 1.63 (95\% Cl 1.04 to 2.55$)$. Intervention participants reported higher clinic satisfaction RR: 2.19 (95\% Cl 1.45 to 3.33). Participants that received the CPR video $(n=110)$ reported greater confidence to perform CPR, RR $1.61(95 \% \mathrm{Cl} 1.20$ to 2.16). Overall, the proportion of participants reporting optimal CV RFs increased between baseline and 30-day follow-up (16.1\% vs $24.8 \%, O R=2.44$ (95\% Cl 1.38 to 4.49)), but there was no significant between-group difference at 30 days.

Conclusion CV education delivery in the waiting room is a scalable concept and may be beneficial to CV care. Larger studies could explore its impact on clinical outcomes.

Trial registration number ANZCTR12618001725257.

\section{INTRODUCTION}

Patient education is an integral aspect of disease management and prevention. Among those with cardiovascular disease (CVD), low health literacy is an independent predictor of mortality. ${ }^{1}$ Furthermore, as poor survival rates from out-of-hospital cardiac arrest (OHCA) gain increased attention, ${ }^{2}$ community education in cardiopulmonary resuscitation (CPR) is becoming an important focus of OHCA management guidelines. ${ }^{3}$ Patients with CVD use healthcare more than the general population, ${ }^{4}$ and for each contact waiting roomtime is often longer than consultation time. ${ }^{5}$ This time could be used to deliver health information, but delivery of generic information is associated with little effect. ${ }^{6}$ Digital health technology presents an opportunity to deliver targeted interventions to patients while they wait, providing information on health conditions of interest and their risk factors.

Video-based educational interventions are feasible to deliver in waiting rooms, potentially scalable to large populations, and there is some evidence supporting their potential health benefit. One randomised clinical trial (RCT) of video-based education delivered to 327 women prior to their antenatal clinic appointment demonstrated a significant improvement in knowledge, exercise, and diet behaviours compared with control. ${ }^{8}$ Yet, another RCT of a stroke educational video delivered to 329 waiting non-acute emergency department patients failed to demonstrate any improvement in CVDrelated lifestyle metrics at 3 months. ${ }^{9}$ Another small $(n=100)$ emergency department-based RCT found participants who watched a 1 min CPR educational video performed CPR better than a control. ${ }^{10}$ To our knowledge, there are no examples of education provision to waiting cardiology patients. This population reports high motivation to make behaviour change, ${ }^{11}$ demonstrates a strong interest in CPR education, ${ }^{12}$ and may be more receptive to receiving CVD-focused education than unselected populations from primary care or the emergency department. Motivation is a key precursor to lifestyle change in educational interventions, ${ }^{13}$ and selfreported confidence to perform CPR is commonly used to describe layperson attitudes towards CPR. ${ }^{14}$

The While You're Waiting study aimed to examine in an RCT the effectiveness of a patientcentred, CVD-focused educational intervention in the waiting room of daily-run Rapid Access Cardiology (RAC) clinics $^{15}$ on motivation to improve CVD-risk modifying behaviours, satisfaction with clinic services and lifestyle behaviours. In a parallel sub-study, we examined the effectiveness of additional CPR-specific education on confidence to perform CPR in the community.

\section{METHODS}

\section{Study design}

While You're Waiting is a single-centre, single-blind, 2:1 parallel designed RCT of patients in the waiting room of a RAC within a tertiary teaching hospital in Sydney, Australia (figure 1). The methods and intervention are described in detail elsewhere. ${ }^{16}$ The 


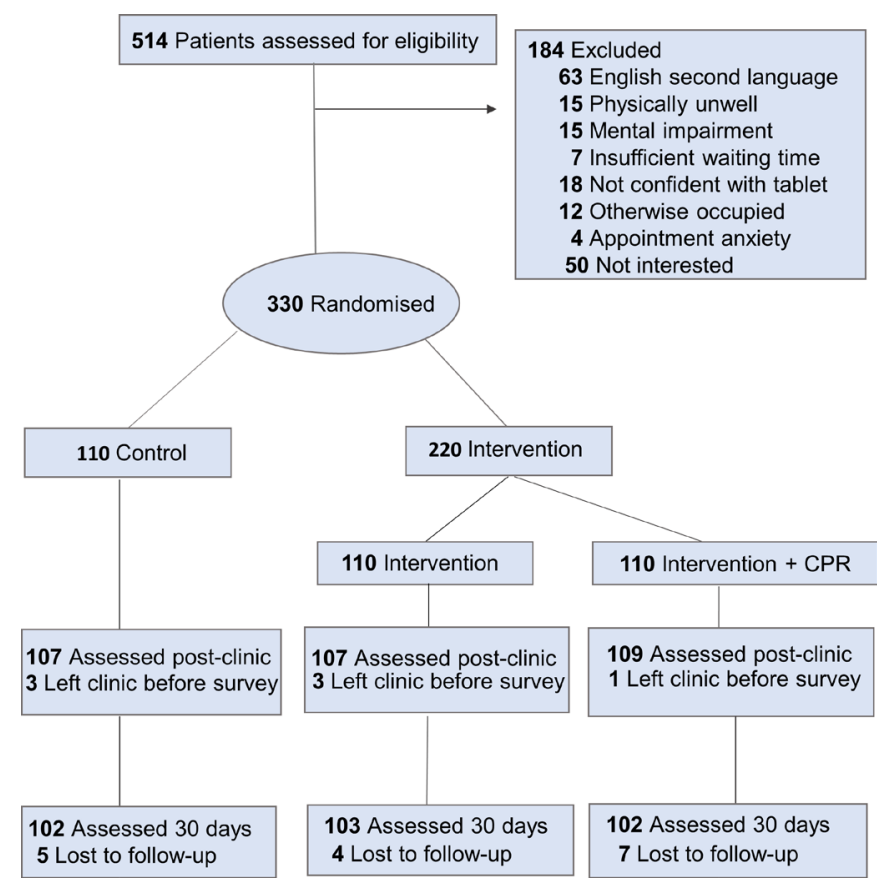

Figure 1 Enrolment of participants in the While You're Waiting Randomised Clinical Trial. CPR, cardiopulmonary resuscitation.

study was delivered via a REDCap survey tool on a computerised tablet device with the support of trained research staff. This integrated consent, randomisation, baseline data collection, and video delivery if the participant was randomised to intervention.

\section{Participants}

The study population was patients presenting to RAC, Westmead Hospital (Sydney, Australia). The RAC is a cardiologist-led, hospital co-located clinic that provides assessment and management of low-intermediate risk patients with suspected coronary heart disease. ${ }^{15}$ Many patients presenting to this clinic have multiple CVD risk factors. ${ }^{17}$ Thus, targeting this patient population with CVD risk factor education presents an opportunity to increase CVD health literacy, lifestyle change, motivation, and ultimately health outcomes.

Patients older than 18 years were eligible. Those too unwell (physically or mentally) to complete surveys and/or watch videos or with insufficient English language competency to provide informed consent were excluded. A log was kept of all participants screened. All participants provided informed consent via an eConsent integrated into the delivery platform.

\section{Patient and public involvement}

The intervention was co-designed with patients, consumers, healthcare providers and researchers and is detailed elsewhere. ${ }^{16}$ Briefly, 21 patients with CVD identified through a consumer organisation contributed to identifying appropriate video content for the intervention programme through rating videos on content utility and likelihood of motivating lifestyle change. Survey data from consumers on CPR confidence informed the sample size calculation for the trial. The 2:1 randomisation design enabled more participants to receive the intervention, and control participants were offered the intervention after the trial. Integration of consent, baseline data collection, intervention delivery and outcome assessment was designed to reduce participant time burden. We plan to disseminate study results to participants following publication.

\section{Randomisation}

Participants were randomised 2:1 (intervention:control). The randomisation sequence was developed by the study statistician (SM) using the Randomise R library of R statistical software (V.3.5.1) in blocks of 3. The intervention allocation sequence was incorporated into RedCap such that treating clinic doctors, recruiting staff and outcome assessors were blinded to patient treatment allocation. Due to constraints of the RedCap randomisation stratification module that requires participants to be randomised on a separate device, impacting both study flow and blinding, the decision was taken to not stratify randomisation according to baseline characteristics.

\section{Intervention programme}

The intervention programme comprised a curated series of cardiovascular risk modification videos delivered on tablet computer devices in the clinic waiting room. Video selection occurred as previously described. ${ }^{16}$ All videos were from reputable sources (including the Australian and British Heart foundations, Healthy WA, and the Better Health Channel) and did not contain advertisements.

Intervention participants were initially asked about their: (1) level of health knowledge (low, medium or high) and (2) topics of interest (physical activity, medications, diet, hypertension, heart attack or general education). According to a pre-specified algorithm, this information was used to select a set of videos for the participant to watch. Videos highly rated by staff and consumers were presented first. It was expected the number of videos watched would vary according to participant preference and length of wait. When the patient was called in for their appointment or they had finished the programme, the tablet was returned to clinic administration staff.

Cardiopulmonary education: a random sub-sample of intervention participants received another video on compressiononly CPR prior to receiving their customised list of CVD videos.

\section{Control}

Following completion of eConsent and baseline surveys on study tablets, control participants proceeded through usual care in the clinic waiting room, which comprised of educational posters/ pamphlets and a small central television.

\section{Trial procedures}

All participants were assessed on three separate occasions:

- Baseline: demographic information, self-reported lifestyle behaviours, CPR confidence (online supplemental appendix 1).

- Immediately post-clinic: motivation to improve diet, exercise and blood pressure monitoring, satisfaction with clinic experience, and CPR confidence, willingness and knowledge (online supplemental appendix 2).

- Thirty-day follow-up: self-reported levels of CV lifestyle risk factors, motivation to improve diet and exercise, CPRrelated items (online supplemental appendix 3).

Baseline clinical and medical history assessment was collected from routine clinical management systems. All other information was collected on study tablets via participant self-report.

\section{Primary outcome (post-clinic)}

Highly motivated to improve CVD-health related behaviours

The primary outcome was defined if a participant responded $\geq 6$ on a 7 -point Likert scale for $\geq 2 / 3$ lifestyle behaviours. Participants reported their agreement with three stem statements: 
'Attending the clinic today motivated me to improve my physical activity/diet/regularly measure my blood pressure'.

\section{Secondary outcomes}

Post-clinic

- Clinic satisfaction: defined if participants reported $\geq 6$ on a 7 -point Likert scale for $\geq 2 / 3$ of three statements on satisfaction: 'I am satisfied with the waiting time/education/overall clinic today'.

- Confidence to perform CPR: defined if participants responded $\geq 4$ on a 5 -point Likert scale of agreement to 'I am confident in my ability to perform CPR'.

-Willingness to perform CPR: defined if participants responded 'yes' to the question 'Would you perform CPR on someone you know/a stranger'.

- CPR knowledge: true/false question: 'To perform CPR, you MUST do breaths AND compressions' (correct answer $=$ false).

Thirty-dayday follow-up

- Lifestyle (exercise and diet) behaviours: defined if participants reported meeting thresholds for $\geq 2 / 3$ measures ( $>30 \mathrm{~min}$ a day for most days of exercise, $>4$ serves of vegetables per day and $>1$ serve of fruit per day). Recommended thresholds were defined according to the National Vascular Disease Prevention Alliance 2012 clinical guidelines. ${ }^{18}$

- Motivation to improve physical activity and diet: defined if participants reported $\geq 6$ on a 7-point Likert scale for 2 stem statements on physical activity and diet, that is, 'I am currently motivated to improve my physical activity/diet'.

- CPR confidence, willingness and knowledge as previously defined.

\section{Statistical considerations}

\section{Study power and sample size}

For the main study, a sample size of 330 had $80 \%$ power, allowing for $5 \%$ attrition, to detect a relative increase of $41 \%$ (RR 1.41) in the proportion of intervention participants reporting high motivation to make lifestyle change. A low attrition rate was assumed as outcomes were collected immediately post-clinic, and a control proportion of $40 \%$ was selected based on evidence that patients with chest pain report high motivation for lifestyle change (mean 6 on a 7 -point Likert scale). ${ }^{11}$

For the sub-study, a sample size of 220 (1:1 intervention:control ratio), with $5 \%$ attrition, had $80 \%$ power to detect a relative increase of $37 \%$ (RR 1.37). A control rate of $50 \%$ was based on data from a survey of CPR confidence administered to 100 visitors entering Westmead Hospital in September 2018. ${ }^{19}$

\section{ANALYTICAL APPROACH}

All analyses were pre-specified in a statistical analysis plan and were conducted according to intention to treat principles. Analyses were performed using $\mathrm{R}$ statistical software (V.3.6.1). All tests were two tailed, a $\mathrm{p}$ value of $<0.05$ was considered significant and relative risks are reported with 95\% CIs. Normally distributed continuous variables were expressed as mean and SD. Non-normally distributed variables were expressed as median and IQR.

The primary analysis for the main and sub-study used a log binomial model adjusted for age, gender, time spent in clinic and educational level. Binary secondary outcomes followed a similar approach. When relevant baseline measures of outcome variables were available (ie, baseline CPR confidence in the CPR substudy), they were also included in statistical models. Primary analysis was a complete-case analysis. Multiple imputation was not pre-specified and hence not used.

Pre-specified analyses of intervention participants who watched $\geq 2$ videos and participants with no previous CPR training were conducted. Exploratory sub-group analyses assessing treatment effect according to age, sex, educational level, body mass index, blood pressure, diabetic status, ethnicity, referral type and waiting time were undertaken.

\section{RESULTS}

Between December 2018 and March 2020, 514 patients attending clinics were screened, 330 randomised (figure 1), 97.8\% (323/330) assessed post-clinic and 93.0\% (307/330) at 30-day follow-up (figure 1). Recruitment closed when the sample size was achieved. Median time in clinic was $115 \mathrm{~min}$ (IQR 90-149). Mean participant age was 53.8 (SD 15.2), 55.2\% $(182 / 330)$ were male and 50.9\% (168/330) had previously completed formal CPR training. The intervention arm reviewed a median of 2.0 (IQR 1.0-4.0) videos, 92.7\% (204/220) watched at least one video and $63.2 \%(139 / 220)$ watched greater than or equal to two videos. Most baseline characteristics were well matched (table 1). Data completeness is included in online supplemental table 1 .

\section{Motivation, satisfaction and lifestyle behaviours}

The primary outcome of high motivation to improve CVD riskmodifying behaviours post-clinic occurred in 29.6\% (64/216) of intervention participants compared with $18.7 \%$ (20/107) of control participants (RR 1.63 (95\% CI 1.04 to 2.55), figure 2). This result was robust to a worst-case analysis for missing data. More intervention participants reported high clinic satisfaction than control participants $(42.1 \%$ (91/216) versus $19.6 \%$ (21/107), RR 2.19 (95\% CI, 1.45, 3.33)). The 139/220 intervention participants who watched $\geq 2$ videos demonstrated a larger difference (figure 2). Between baseline and 30 days, the proportion of all participants reporting achieving $\geq 2 / 3$ diet and physical activity thresholds increased $(16.1 \%$ vs $24.8 \%, \mathrm{OR}=2.44(95 \%$ CI 1.38 to 4.49 ), see figure 2); however, there was no significant difference between groups at 30 days (figure 2, table 2).

\section{CPR confidence, willingness and knowledge among CPR video recipients}

Post-clinic, self-reported confidence to perform CPR was achieved in $57.8 \%(63 / 109)$ of participants that viewed the CPR video compared with 36.4\% (39/107) of control participants (RR 1.61 (95\% CI 1.20 to 2.16), figure 2). There was a greater difference among the $162 / 330$ participants with no prior CPR training $(45.8 \%$ (22/49) confident vs control - $16.0 \%$ (8/53) confident, RR 2.50 (95\% CI 1.24 to 5.04)). Willingness to perform CPR on someone they knew was higher in those that received the CPR video compared with control (RR 1.36 (95\% CI 1.15 to 1.62 ), and willingness to perform CPR on a stranger was also higher (RR 1.43 (95\% CI 1.18 to 1.72$)$ ). CPR video recipients were more likely to correctly answer the CPR knowledge question (RR 3.80 (95\% CI 2.13 to 6.77)).

At 30 days, the difference in CPR knowledge was maintained (39.8\% (41/103) vs $14.7 \%$ (15/102), RR 2.72 (95\% CI 1.60 to 4.64). Differences in confidence and willingness no longer reached significance (RR 1.25 (95\% CI 0.97 to 1.60$)$, RR 1.10 (95\% CI 0.94 to 1.29 ) for someone known, RR 1.14 (95\% CI 0.95 to 1.35 ) for a stranger (figure 2)). 
Table 1 Baseline demographic characteristics

\begin{tabular}{|c|c|c|c|c|c|}
\hline Baseline characteristics & $\begin{array}{l}\text { Control } \\
(n=110)\end{array}$ & $\begin{array}{l}\text { Intervention total } \\
(\mathrm{n}=220)\end{array}$ & $\begin{array}{l}\text { Additional CPR video } \\
(\mathrm{n}=110)\end{array}$ & $\begin{array}{l}\text { No CPR video } \\
(n=110)\end{array}$ & $\begin{array}{l}\text { Total } \\
(n=330)\end{array}$ \\
\hline Age, mean (SD) & $53.0( \pm 15.0)$ & $54.2( \pm 15.4)$ & $54.1( \pm 15.2)$ & $54.2( \pm 15.6)$ & $53.8( \pm 15.2)$ \\
\hline Male, n (\%) & 70 (63.6) & $112(50.9)$ & $56(50.9)$ & $56(50.9)$ & $182(55.2)$ \\
\hline \multicolumn{6}{|l|}{ Ethnicity, n (\%) } \\
\hline Aboriginal/Torres Strait Islander & $2(1.8)$ & $2(0.9)$ & $1(0.9)$ & $1(0.9)$ & $4(1.2)$ \\
\hline European & $39(35.5)$ & $83(37.7)$ & 37 (33.6) & $46(41.8)$ & $122(37.0)$ \\
\hline Asian (South, East, South-East) & $38(34.5)$ & $65(29.5)$ & $34(30.9)$ & $31(28.2)$ & $103(31.2)$ \\
\hline Middle East and North African & $10(9.1)$ & $27(12.3)$ & $12(10.9)$ & $15(13.6)$ & $37(11.2)$ \\
\hline Other & $21(19.1)$ & $43(19.5)$ & $26(23.6)$ & $17(15.5)$ & $64(19.4)$ \\
\hline \multicolumn{6}{|l|}{ Education, n (\%) } \\
\hline Completed tertiary qualification & $56(50.9)$ & $115(52.3)$ & $66(60.0)$ & $49(44.5)$ & $171(51.8)$ \\
\hline Confident ${ }^{*}$ to perform CPR & $43(39.1)$ & $80(36.4)$ & $42(38.2)$ & $38(34.5)$ & $123(37.3)$ \\
\hline Completed formal CPR training & $57(51.8)$ & $111(50.5)$ & $61(55.5)$ & $50(45.5)$ & $168(50.9)$ \\
\hline Total time spent in clinic, median time (IQR) & $120.0(91.5-152.2)$ & $113.0(89.5-144.5)$ & $117.0(86.0-140.0)$ & $110.0(90.0-149.5)$ & $115.0(90.0-149.0)$ \\
\hline \multicolumn{6}{|l|}{ Number of videos watched } \\
\hline Median videos (IQR) & - & $2.0(1.0-4.0)$ & $2.0(1.0-4.0)$ & $2.0(1.0-6.0)$ & $1.0(1.0-3.0)$ \\
\hline \multicolumn{6}{|l|}{ Reason for referral, $\mathrm{n}(\%)$} \\
\hline Typical chest pain & $16(14.5)$ & $45(20.5)$ & $24(21.8)$ & $21(19.1)$ & $61(18.5)$ \\
\hline Atypical chest pain & $57(51.8)$ & $83(37.7)$ & $37(33.6)$ & $46(41.8)$ & $140(42.4)$ \\
\hline Arrhythmia & $16(14.5)$ & $38(17.3)$ & $21(19.1)$ & $17(15.5)$ & $54(16.4)$ \\
\hline Syncope & $6(5.5)$ & $11(5.0)$ & $7(6.4)$ & $4(3.6)$ & $17(5.2)$ \\
\hline Heart failure evaluation & $4(3.6)$ & $9(4.1)$ & $5(4.5)$ & $4(3.6)$ & $13(3.9)$ \\
\hline Other & $9(8.2)$ & $17(7.7)$ & $9(8.2)$ & $8(7.3)$ & $26(7.9)$ \\
\hline \multicolumn{6}{|l|}{ Lifestyle - self-reported, n (\%) } \\
\hline Diabetes & $14(12.7)$ & $54(24.5)$ & $27(24.5)$ & $27(24.5)$ & $68(20.6)$ \\
\hline Hyperlipidaemia & $56(50.9)$ & $98(44.5)$ & $54(49.1)$ & $44(40.0)$ & $154(46.7)$ \\
\hline Hypertension & $46(41.8)$ & $103(46.8)$ & $51(46.4)$ & $52(47.3)$ & $149(45.2)$ \\
\hline Existing coronary heart disease & $16(14.5)$ & $40(18.2)$ & $20(18.2)$ & $20(18.2)$ & $56(17.0)$ \\
\hline Family history of coronary heart disease & $43(39.1)$ & $84(38.2)$ & $41(37.3)$ & $43(39.1)$ & $127(38.5)$ \\
\hline Current smoker & $20(18.2)$ & $41(18.6)$ & $18(16.4)$ & $23(20.9)$ & $61(18.5)$ \\
\hline 30 min exercise on most days or greater & $52(47.2)$ & $86(39.0)$ & $37(33.6)$ & $49(44.5)$ & $138(41.8)$ \\
\hline $\begin{array}{l}\text { Greater than four serves of vegetables per } \\
\text { day }\end{array}$ & $13(11.8)$ & $14(6.4)$ & $9(8.2)$ & $5(4.5)$ & $27(8.2)$ \\
\hline Greater than one serve of fruit per day & $65(59.1)$ & $117(53.2)$ & $56(50.9)$ & $61(55.5)$ & $182(55.2)$ \\
\hline \multicolumn{6}{|l|}{ Clinical assessment } \\
\hline Height $(\mathrm{cm})$ & $169.3( \pm 9.2)$ & $167.6( \pm 10.0)$ & $168.2( \pm 9.5)$ & $167.1( \pm 10.5)$ & $168.2( \pm 9.7)$ \\
\hline Weight (kg) & $85.4( \pm 20.3)$ & $83.3( \pm 20.2)$ & $82.1( \pm 18.6)$ & $84.6( \pm 21.6)$ & $84.0( \pm 20.2)$ \\
\hline BMI $\left(\mathrm{kg} / \mathrm{m}^{2}\right)$ & $29.6( \pm 6.0)$ & $29.6( \pm 6.5)$ & $29.1( \pm 6.4)$ & $30.2( \pm 6.5)$ & $29.6( \pm 6.3)$ \\
\hline $\mathrm{SBP}(\mathrm{mm} \mathrm{Hg})$ & $128.4( \pm 18.0)$ & $130.9( \pm 18.3)$ & $130.2( \pm 19.6)$ & $131.6( \pm 16.9)$ & $130.1( \pm 18.2)$ \\
\hline $\mathrm{DBP}(\mathrm{mm} \mathrm{Hg})$ & $79.1( \pm 9.5)$ & $79.4( \pm 10.4)$ & $78.6( \pm 10.2)$ & $80.1( \pm 10.5)$ & $79.3( \pm 10.1)$ \\
\hline
\end{tabular}

Confident to perform CPR $=$ self-reported confidence $\geq 4 / 5$.

BMI, body mass index; DBP, diastolic blood pressure; SBP, systolic blood pressure.

\section{Ancillary analyses}

Exploratory subgroup analyses are shown in figure 3. Heterogeneity of effect of the study intervention on the primary outcome was suggested according to gender $(p=0.012)$. No other significant subgroup interactions were identified for the primary outcome or the main outcome of the CPR sub-study.

\section{DISCUSSION}

It is well-known that positive lifestyle change reduces CVDrelated mortality. ${ }^{20}$ This RCT found a patient-centred, tablet-delivered educational programme on cardiovascular risk significantly increased motivation to improve CVD riskmodifying behaviours and clinic satisfaction (figure 4). Provision of an additional brief CPR video significantly increased confidence, knowledge and willingness to perform CPR post-clinic.
Overall, the proportion of participants reporting achieving diet and exercise thresholds was significantly higher at 30-day follow-up than baseline (figure 2), though the difference between groups did not reach statistical significance. Differences in other key outcomes also attenuated and became non-significant at 30-day follow-up. Additional analyses examining intervention participants who watched $\geq 2$ videos suggest the intervention may be more effective in patients who were more engaged with the intervention (figure 2). Ancillary subgroup analysis suggests females were more likely to achieve the primary outcome than males. This may be due to differences in healthcare attitudes, intervention material and delivery, or chance. Further research to explore reasons for intervention effectiveness among different patient populations is needed. 

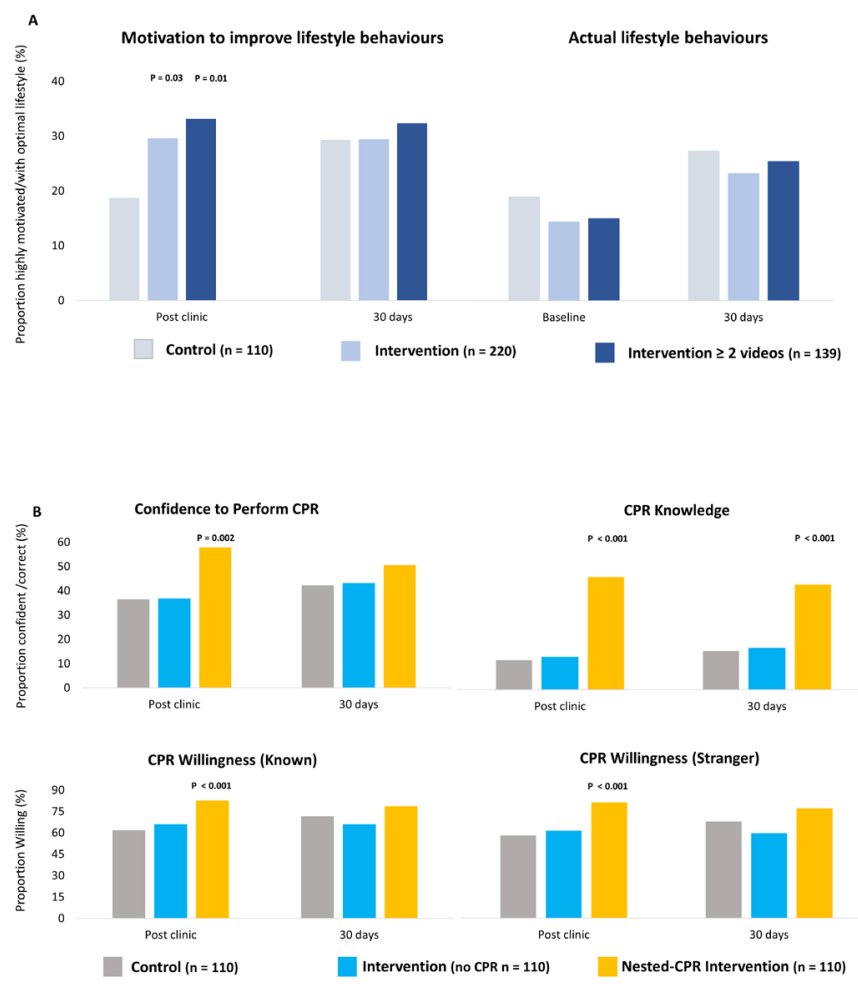

Intervention (no CPR $\mathrm{n}=110$ )

Nested-CPR Intervention ( $\mathrm{n}=110$ )

Figure 2 (A) Participant motivation and lifestyle behaviours post-clinic and at 30-day follow-up. (B) CPR confidence, willingness and knowledge post-clinic and 30 days all outcomes adjusted for age, gender, education and total waiting time. ${ }^{*} \mathrm{P}<0.05,{ }^{*} \mathrm{p}<0.01$. All $p$ values are relative to control participants unless stated otherwise.

Our literature review identified no similar RCTs of educational interventions in cardiology clinic waiting rooms. However, study results are broadly consistent with non-randomised emergency department-based studies

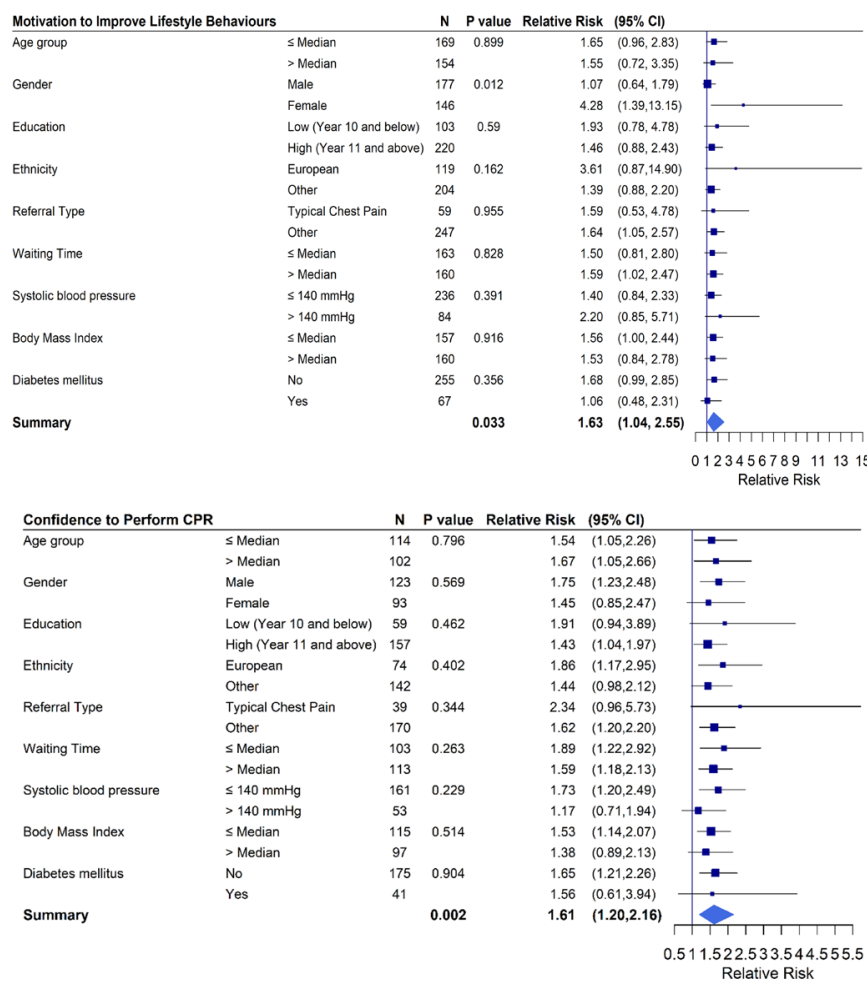

Figure 3 Subgroup analyses of primary outcomes. Based on age, gender, education, ethnicity, referral type, waiting time, blood pressure, body mass index and diabetic status. P value describes whether intervention effect differs between subgroups for the two primary outcomes.

demonstrating waiting room education improved health service satisfaction. $^{21} 22$

Multiple studies have shown that exposure to CPR videos can improve CPR performance ${ }^{1023}$ and confidence to perform CPR. ${ }^{24}$

Table 2 Primary and secondary analyses and sensitivity analysis restricted to participants who watched $\geq 2$ videos

$\begin{array}{llllll}\text { Control } & \text { Intervention }(\mathrm{n}=216 \text { post-clinic }(139 & & & \\ \text { ( } \mathrm{n}=107 \text { post-clinic, } 102 & \geq 2 \text { videos)), } 205 \text { at } 30 \text { days }(133 & \text { Relative risk } & & \text { Relative risk } \\ \text { at } 30 \text { days) }(\mathrm{n}(\%)) & \geq 2 \text { videos) }(\mathrm{n}(\%)) & \begin{array}{l}\text { (unadjusted) } \\ \text { (adjusted) }\end{array} & \text { P value } & \text { P value }\end{array}$

\section{Post-clinic}

Primary outcome

Motivation to improve health behaviours (exercise, diet and blood pressure monitoring)

\begin{tabular}{|c|c|c|c|c|c|c|}
\hline Highly* motivated & $20(18.7)$ & $64(29.6)$ & 1.59 (1.02 to 2.47$)$ & 0.04 & 1.63 (1.04 to 2.55$)$ & 0.03 \\
\hline$\geq 2$ videos & $20(18.7)$ & $46(33.1)$ & 1.77 (1.12 to 2.81$)$ & 0.01 & 1.90 (1.20 to 3.00$)$ & 0.01 \\
\hline \multicolumn{7}{|c|}{ Secondary outcomes } \\
\hline \multicolumn{7}{|c|}{ Satisfaction with clinic experience (waiting time, education and clinic overall) } \\
\hline Highly* satisfied & $21(19.6)$ & $91(42.1)$ & 2.15 (1.42 to 3.25$)$ & $<0.001$ & $2.19(1.45$ to 3.33$)$ & $<0.001$ \\
\hline$\geq 2$ videos & $21(19.6)$ & $62(44.6)$ & 2.27 (1.48 to 3.48$)$ & $<0.001$ & 2.35 (1.53 to 3.60$)$ & $<0.001$ \\
\hline \multicolumn{7}{|c|}{ Follow-up (30 days) } \\
\hline \multicolumn{7}{|c|}{ Motivation to improve exercise and diet only } \\
\hline Highly motivatedt & $30(29.4)$ & $60(29.3)$ & $1.0(0.69$ to 1.44$)$ & $>0.9$ & 0.99 (0.69 to 1.43$)$ & $>0.9$ \\
\hline$\geq 2$ videos & $30(29.4)$ & $43(32.3)$ & 1.1 (0.75 to 1.62$)$ & 0.63 & 1.07 (0.73 to 1.58$)$ & 0.72 \\
\hline \multicolumn{7}{|c|}{ Actual lifestyle behaviours (exercise, vegetable intake and fruit intake) } \\
\hline Optimal‡ lifestyle & $28(27.5)$ & $48(23.4)$ & $1.0(0.71$ to 1.40$)$ & $>0.9$ & $0.82(0.55$ to 1.23$)$ & 0.33 \\
\hline$\geq 2$ videos & $28(27.5)$ & $34(25.6)$ & 0.98 (0.66 to 1.43$)$ & $>0.9$ & 0.92 (0.60 to 1.41$)$ & 0.70 \\
\hline
\end{tabular}

Bold values are statistically significant.

*Highly motivated/satisfied $=\geq 6 / 7$ for $\geq 2 / 3$ of lifestyle behaviours/satisfaction measures.

tHighly motivated $=\geq 6 / 7$ for $2 / 2$ lifestyle behaviours.

$\ddagger$ Optimal = self-reported lifestyle behaviours achieving guideline indicated thresholds for $\geq 2 / 3$ of exercise, vegetable intake and fruit intake. All measures adjusted for age, gender, educational level and total time in clinic. 


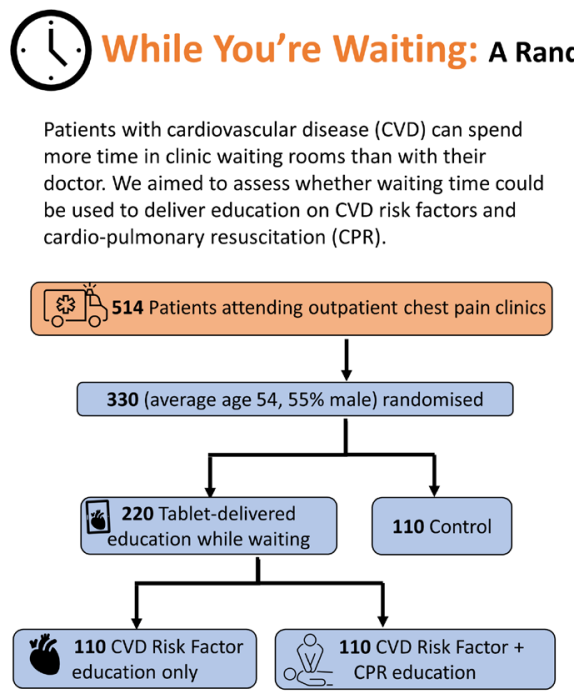

Figure 4 Informational graphic summary of the While You're Waiting study.
After clinic, participants who had received the program were more likely to report:

This concept is feasible and scalable. Future studies should examine the potential for impact on clinical outcomes

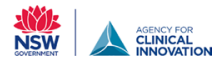

A recent systematic review found that community focused CPR interventions improve 30-day survival from OHCA (OR 1.34; $95 \%$ CI 1.14 to 1.57$).{ }^{25}$ Waiting room delivery of CPR education is a novel approach with substantial reach and is likely to be easily scaled. Our study demonstrates delivering CPR education in the clinic waiting room is feasible and effective in improving confidence and willingness to perform CPR. The sub-analysis among participants with no previous CPR training indicated intervention impact may be higher within this group suggesting that this type of intervention may be particularly useful in areas of low health literacy or low knowledge of CPR.

\section{Clinical implications}

As pressure on public health systems mounts and patients wait longer, ${ }^{26}$ interventions of this nature could contribute to patient care in various healthcare settings. Educational interventions such as this are low cost and while their effect sizes on direct health outcomes are likely to be small, they are amenable to provision at scale. As such, the observed effect would need not be large to justify scaled implementation. Using time otherwise not used effectively during patient care makes good clinical sense, and if it empowers patients to understand and self-manage their condition is likely to have additional health and economic benefits.

The large immediate intervention effect on motivation shown in our study could also be harnessed to engage patients in longer term behaviour change interventions. Taking the opportunity of the clinic visit to commence them on this journey is a potentially useful opportunistic intervention. This could be facilitated through extended access to videos and/or the delivery of mobile health strategies that have shown promise in primary and secondary prevention settings. ${ }^{27} 28$ Delivered at a population level, the intervention may offer a cost-effective approach to provision of CVD and chronic disease preventative programmes.

\section{Limitations}

There are several limitations to this study. First, for brevity for participants in a waiting room, we designed a short survey to measure study outcomes we thought relevant from our review of the literature. We piloted this questionnaire among consumers prior to the study but did not carry out formal validity or reliability assessments. The positive wording of some questions may have biased participants to respond positively, though this effect would be experienced equally by intervention and control participants. Second, this is a relatively small, single-centre study and findings may not be generalisable to other patient populations or powered to reach conclusions on small changes to CVDrelated lifestyle behaviours. Third, it was not possible to achieve complete blinding in this study. We did take measures to prevent selection biases and blind study staff (eg, by providing tablets initially to both intervention and control participants). However, a Hawthorne effect may have influenced study results postclinic. Finally, small differences were observed in some baseline

\section{Key messages}

What is already known on this subject?

- Waiting time is ubiquitous in healthcare but poorly used.

- There are no examples of randomised clinical trials assessing the impact of waiting room intervention delivery on cardiology patients.

What might this study add?

- In this randomised clinical trial of 330 adults, participants who received waiting room education were 1.6 times more likely to report high motivation to improve cardiovascular lifestyle behaviours than control participants post-clinic.

- A sub-set of 110 participants who received education on cardiopulmonary resuscitation (CPR) were 1.6 times more likely to report high confidence to perform CPR than control participants post-clinic.

- At 30 days, there was no significant impact on actual lifestyle behaviours, though the study was not powered for this outcome.

How might this impact on clinical practice?

- Provision of targeted waiting room education improves patient engagement with cardiovascular disease prevention and could be a feasible and low-cost vehicle for CPR education.

- Larger, multi-centre studies are needed to determine the effect on clinical outcomes. 
characteristics between intervention and control groups. This was likely due to chance in a relatively small sample size study without stratification. Post-hoc analysis of the randomisation algorithm (10000 iterations with the same sequence of males and females observed in the study) indicated that, without stratification, the likelihood of a $\geq 12.7 \%$ difference in proportion of male participants, as observed, is $4.2 \%$.

\section{CONCLUSION}

Waiting time is increasing, underused and presents an opportunity for the delivery of interventions to improve patient engagement and health outcomes. Our study demonstrated that a simple waiting room-based educational programme had a large immediate impact on motivation to improve CVD risk-modifying behaviours and confidence to perform CPR. The study and concept are scalable and could easily be delivered to larger populations with little risk and the potential for significant population level benefit. Future studies should examine whether sustained intervention deilvery outside the waiting room is feasible. There is also a need for larger, multi-centre waiting room-based studies powered to assess the potential for population level impact on CVD risk and outcomes.

\section{Twitter Clara K Chow @clara_chow}

Acknowledgements We acknowledge Robert Reid, secretary of the Cardiomyopathy Association of Australia, for his contribution to the development of the intervention. We would also like to thank staff of the Rapid Access Cardiology Clinic, Westmead Hospital and the Westmead Applied Research Centre for their support.

Contributors Study concept and design: DM, AT and CKC. Acquisition, analysis or interpretation of data: all authors. Drafting of manuscript: DM, AT and CKC. Critical revision of manuscript: all authors. Statistical analysis: DM, AVH, SM and CKC. Obtained funding: DM, AT and CKC. Study supervision: AT and CKC.

Funding This work was supported by the Agency for Clinical Innovation Research Grants Scheme. CKC is supported by a National Health and Medical Research Council of Australia Career Development Fellowship cosponsored by the National Heart Foundation of Australia.

\section{Competing interests None declared.}

Patient consent for publication Not required.

Ethics approval Ethical approval was obtained from the Western Sydney Local Health District Human Research Ethics Committee (5621) AU RED HREC/18/ WMEAD/124

Provenance and peer review Not commissioned; internally peer reviewed.

Data availability statement Data are available on reasonable request. Deidentified study data will be made available to researchers who provide a methodologically sound proposal and after the signing of a non-disclosure agreement. To submit a data use proposal, please email the principal investigator at clara.chow@sydney.edu.au.

Supplemental material This content has been supplied by the author(s). It has not been vetted by BMJ Publishing Group Limited (BMJ) and may not have been peer-reviewed. Any opinions or recommendations discussed are solely those of the author(s) and are not endorsed by BMJ. BMJ disclaims all liability and responsibility arising from any reliance placed on the content. Where the content includes any translated material, BMJ does not warrant the accuracy and reliability of the translations (including but not limited to local regulations, clinical guidelines, terminology, drug names and drug dosages), and is not responsible for any error and/or omissions arising from translation and adaptation or otherwise.

\section{ORCID iDs}

Daniel McIntyre http://orcid.org/0000-0003-4854-4050

Simone Marschner http://orcid.org/0000-0002-5484-9144

Clara K Chow http://orcid.org/0000-0003-4693-0038

\section{REFERENCES}

1 McNaughton CD, Cawthon C, Kripalani S, et al. Health literacy and mortality: a cohort study of patients hospitalized for acute heart failure. J Am Heart Assoc 2015;4:e01799

2 The Lancet. Out-of-hospital cardiac arrest: a unique medical emergency. The Lancet 2018;391:911

3 Cheng A, Nadkarni VM, Mancini MB, et al. Resuscitation education science: educational strategies to improve outcomes from cardiac arrest: a scientific statement from the American heart association. Circulation 2018;138:e82-122.

4 Bähler C, Huber CA, Brüngger B, et al. Multimorbidity, health care utilization and costs in an elderly community-dwelling population: a claims data based observational study. BMC Health Serv Res 2015;15:23.

5 Patwardhan A, Davis J, Murphy P, et al. Comparison of waiting and consultation times in convenient care clinics and physician offices: a cross-sectional study. J Prim Care Community Health 2013:4:124-8.

6 Gignon M, Idris $\mathrm{H}$, Manaouil C, et al. The waiting room: vector for health education? the general practitioner's point of view. BMC Res Notes 2012;5:511.

7 Warner L, Klausner JD, Rietmeijer CA, et al. Effect of a brief video intervention on incident infection among patients attending sexually transmitted disease clinics. PLoS Med 2008:5:e135.

8 Jackson RA, Stotland NE, Caughey AB, et al. Improving diet and exercise in pregnancy with video doctor counseling: a randomized trial. Patient Educ Couns 2011;83:203-9.

9 Yvonne Chan Y-F, Nagurka R, Richardson LD, et al. Effectiveness of stroke education in the emergency department waiting room. J Stroke Cerebrovasc Dis 2010;19:209-15.

10 Benoit JL, Vogele J, Hart KW, et al. Passive ultra-brief video training improves performance of compression-only cardiopulmonary resuscitation. Resuscitation 2017:115:116-9.

11 Deci EL, Gagné M, Mushlin Al. Motivation for behavior change in patients with chest pain. Health Education 2005;105:304-21.

12 Cartledge S, Feldman S, Bray JE, et al. Understanding patients and spouses experiences of patient education following a cardiac event and eliciting attitudes and preferences towards incorporating cardiopulmonary resuscitation training: a qualitative study. J Adv Nurs 2018;74:1157-69.

13 Hou S-I. Health education: theoretical concepts, effective strategies and core competencies. Health Promot Pract 2014;15:619-21.

14 Cartledge S, Saxton D, Finn J, et al. Australia's awareness of cardiac arrest and rates of CPR training: results from the Heart Foundation's HeartWatch survey. BMJ Open 2020;10:e033722.

15 Klimis H, Khan ME, Thiagalingam A, et al. Rapid access cardiology (Rac) services within a large tertiary referral Centre-First year in review. Heart Lung Circ 2018;27:1381-7.

16 Mcintyre D, Thiagalingam A, Chow C. While You're Waiting, a waiting room-based, cardiovascular disease-focused educational program: protocol for a randomised controlled trial. BMJ Open 2020;10:e036780.

17 Klimis $\mathrm{H}$, Khan ME, Thiagalingam A, et al. Rapid access cardiology (Rac) services within a large tertiary referral Centre-First year in review. Heart, Lung and Circulation 2018;27:1381-7.

18 Alliance NVDP. Clinical guideline for the management of absolute cardiovascular disease risk, 2012.

19 Czinner T. Press release: WARC and partners celebrate World Heart Day, 2018. Available: https://www.sydney.edu.au/content/dam/corporate/documents/research/ WHF-warc-press-release_V4.pdf

20 Anderson L, Oldridge N, Thompson DR, et al. Exercise-Based cardiac rehabilitation for coronary heart disease: cochrane systematic review and meta-analysis. J Am Coll Cardiol 2016;67:1-12.

21 Papa L, Seaberg DC, Rees E, et al. Does a waiting room video about what to expect during an emergency department visit improve patient satisfaction? CJEM 2008; 10:347-54.

22 Reid S, Neto G, Tse S, et al. Education in the waiting room: description of a pediatric emergency department educational initiative. Pediatr Emerg Care 2017:33:e87-91.

23 Bobrow BJ, Vadeboncoeur TF, Spaite DW, et al. The effectiveness of Ultrabrief and brief educational Videos for training lay responders in Hands-Only cardiopulmonary resuscitation. Circulation 2011;4:220-6.

24 Lynch B, Einspruch EL. With or without an instructor, brief exposure to CPR training produces significant attitude change. Resuscitation 2010;81:568-75.

25 Yu Y, Meng Q, Munot S, et al. Assessment of community interventions for bystander cardiopulmonary resuscitation in out-of-hospital cardiac arrest: a systematic review and meta-analysis. JAMA Netw Open 2020;3:e209256.

26 lacobucci G. Waiting list for elective surgery tops four million for first time since 2007. BMJ 2017;358:j3861.

27 Chow CK, Redfern J, Hillis GS, et al. Effect of Lifestyle-Focused text messaging on risk factor modification in patients with coronary heart disease: a randomized clinical trial. JAMA 2015;314:1255-63.

28 Klimis H, Thakkar J, Chow CK. Breaking barriers: mobile health interventions for cardiovascular disease. Can J Cardiol 2018;34:905-13. 